\title{
A Retrospective Study on Antibiotic Microbial Sensitivity in Type II Diabetes Mellitus Patients with Urinary Tract Infections
}

\author{
Anitha Kuttiappan', Sasi Prasad Dondapati ${ }^{2, *}$, Tejasri Kothinti ${ }^{2}$, Manoja Bayyapureddy ${ }^{2}$, Jyosthna Kothapalli², Anna Balaji ${ }^{3}$ \\ 'Department of Pharmacology, Sree Vidyanikethan College of Pharmacy, Tirupati, Andhra Pradesh, INDIA. \\ 2Department of Pharmacy Practice, Sree Vidyanikethan College of Pharmacy, Tirupati, Andhra Pradesh, INDIA. \\ ${ }^{3}$ Department of Pharmaceutics, Sree Vidyanikethan College of Pharmacy, Tirupati, Andhra Pradesh, INDIA.
}

\begin{abstract}
Objectives: The present study was aimed to observe the antibiotic microbial sensitivity patterns in type II Diabetes Mellitus patients with Urinary tract infections. Methods : A retrospective study was conducted in the Department of Endocrinology and Metabolism at Sri Venkateswara Institute of Medical Sciences, Tirupati during a period of 6 months (July to December 2019). 120 culture urine samples were taken for the study as per inclusion and exclusion criteria. All the data were collected, recorded, tabulated and analysed using Microsoft Excel worksheet (Microsoft Corp, Redmond, WA). Continuous and categorical data were expressed in terms of mean \pm Standard deviation (SD) and percentage respectively. Antibiotic microbial sensitivity was expressed in terms of percentage. Results: Out of the total of 120 subjects, the most commonly affected age group was $51-$ 60 years $(33.33 \%)$. Here females $(55 \%)$ dominated males $(45 \%)$ in number. Most commonly isolated Gram-negative organisms were Escherichia coli $(35 \%)$ and Klebsiella pneumoniae (15.8\%) whereas Gram-positive organisms were Enterococcus faecalis (17.6\%) and Staphylococcus aureus (9.16\%). Most of the gram-negative bacteria were having a good sensitivity to amikacin $(56.75 \%)$ and gram-positive bacteria were mostly sensitive to nitrofurantoin (46\%). Conclusion: Escherichia coli was the major isolated
\end{abstract}

micro-organism followed by Enterococcus and Klebsiella. Amikacin showed more sensitivity towards gram-negative organisms, whereas Nitrofurantoin showed more sensitivity towards gram-positive organisms. Most uropathogens isolated in Type II Diabetes mellitus were sensitive to Amikacin. This study enlists certain antibiotics which can be used as firstline agents by the physician while awaiting for urine microscopic culture sensitivity results.

Key words: Diabetes Mellitus, Retrospective Study, Urinary Tract Infection, Antibiotic Sensitivity, Gram-Positive Organisms, Gram-Negative Organisms.

Correspondence

Dr. Sasi Prasad Dondapati

Department of Pharmacy Practice, Sree Vidyanikethan College of Pharmacy, Tirupati, Andhra Pradesh, INDIA.

Phone: +91-7780589005

Email: dondapatisasiprasad97@gmail.com

DOI: 10.5530/jyp.2021.13.14

\section{INTRODUCTION}

Diabetes mellitus (DM) is a worldwide health problem with dramatically raised prevalence over the past two decades and expected prevalence of 593 million by $2035 .{ }^{1}$ Over 425 million people are currently living with DM. Based on the report of the International Diabetes Federation (IDF), China has the largest number of diabetic cases (11.43cr.), followed by India $(7.29 \mathrm{cr}$.) in 2017 . The prevalence of type II diabetes mellitus in number of adults has increased almost four times in less than four decades from 108 million in 1980 to 422 million. Diabetes mellitus is a group of metabolic diseases characterised by hyperglycaemia resulting from defects in insulin secretion, insulin action or both. The chronic hyperglycaemia of diabetes is associated with long term damage, dysfunction and failure of different organs especially the eyes, kidneys, nerves, heart and blood vessels. Diabetes mellitus is a major predisposing factor for the development of Urinary Tract Infection (UTI) as it modifies the host defence mechanism, Immunologic impairments, such as defective in migration and phagocytic properties of polymorph nuclear leucocytes were occurred in diabetes mellitus patients. Urinary tract infection (UTI) is the most common infection among patients with $\mathrm{DM}$ and is responsible for considerable morbidity particularly if it is unrecognised or untreated. Patients with type II DM are at increased risk of urinary tract infection being the most common frequent site. ${ }^{2,3}$ Bacteriuria is more common in diabetics than in non-diabetics due to a combination of host and local risk factors.
The patients with urinary tract infections varies from asymptomatic Bacteriuria to lower UTI (cystitis), pyelonephritis and severe urosepsis. The complications of Urinary tract infections such as emphysematous cystitis and pyelonephritis, renal abscesses and renal papillary necrosis were all encountered more frequently in type II diabetes mellitus patients than in the general population. Type II DM is not only the risk factor for community-acquired UTI but also for health-care associated UTI, catheter associated UTI, post renal transplant-recurrent UTI. Type II DM is also a risk factor for fungal UTI, mostly caused by Candida. The increased risk of UTI among diabetic patients, coupled with the increase in the incidence of type II DM worldwide. ${ }^{4}$

The diagnosis of urinary tract infections should be suspected in any diabetic patient with symptoms consistent with urinary tract infections. These symptoms are frequency, urgency, dysuria and supra-pubic pain for lower urinary tract infections and costo-vertebral angle pain/tenderness, fever and chills, with or without lower urinary tract symptoms for upper urinary tract infections. ${ }^{5}$ Diabetic patients are prone to have a more severe presentation of urinary tract infections though some patients with diabetic neuropathy may have altered clinical signs. Once the diagnosis of urinary tract infections is suspected, a midstream urine specimen should be examined for the presence of leukocytes, as pyuria is present in almost all cases of urinary tract infections. ${ }^{6}$

This is an open access article distributed under the terms of the Creative Commons Attribution-NonCommercial-ShareAlike 4.0 License, which allows others to remix, tweak, and build upon the work non-commercially, as long as the author is credited and the new creations are licensed under the identical terms. 
Escherichia coli, Klebsiella pneumoniae, Enterobacter aerogenes, Proteus mirabilis, Enterococcus faecalis, Staphylococcus aureus and Pseudomonas aeruginosa were the most common etiological agents causing UTI. ${ }^{78}$ The current study aims to assess the causative organisms and the antibiotic sensitivity patterns in type II diabetes mellitus with UTI.

\section{MATERIALS AND METHODS}

\section{Study design and Ethical consideration}

A Retrospective Study conducted on Antibiotic Microbial Sensitivity in Type II Diabetes Mellitus Patients with Urinary Tract Infections in the Department of Endocrinology and Metabolism at Sri Venkateswara Institute of Medical Sciences, Tirupati, Andhra Pradesh, India in 2019. The ethical clearance was obtained from the Institutional Ethics Committee with approved IEC NO. 920 from Sri Venkateswara Institute of Medical Sciences. The study was performed as per ICH GCP Guidelines.

\section{Selection criteria}

Patients who were diagnosed as type II Diabetes Mellitus with Urinary Tract Infections and whose pus cells count $>8 \mathrm{hpf}$ (high power field) were taken as inclusion criteria. Urinary tract infections in non-diabetic patients, Pregnant and Lactation women were excluded.

\section{Sample size}

One hundred and twenty patients who were diagnosed as type II Diabetes mellitus with UTI were taken as sample size and examined as per study guidelines.

\section{Method of Collection of Data}

The patient case reports were taken from the medical records department based on inclusion and exclusion criteria. Data regarding isolated organisms were collected. All the necessary parameters like baseline characteristics (age, gender, duration of DM, systolic Blood pressure, Diastolic blood pressure, $\mathrm{HbA}_{1 \mathrm{c}}$, duration of fever, duration of hospital stay) and clinical manifestations (fever, vomiting, dysuria, abdominal pain, lower backache, frequent urination) were recorded .we also collected all the necessary information to evaluate the microbial sensitivity with respect to the antibiotics.

\section{Statistical analysis}

Data was recorded on a predesigned proforma and managed using Microsoft Excel worksheet (Microsoft Corp, Redmond, WA). Data entered were double-checked for any possible errors. Continuous data were expressed in terms of mean \pm Standard deviation (SD). Categorical data were expressed in terms of percentage. Antibiotic microbial sensitivity was expressed in terms of percentage. Data were analysed according to the objectives of the study.

\section{RESULTS}

This study was attempted to evaluate the isolated organisms, antibiotic microbial sensitivity pattern in type II diabetes mellitus patients with urinary tract infections. The study included 120 patients who came to the endocrinology department.

\section{Distribution of data based on age group}

As per study among the 120 subjects, majority of affected patients were in the age group of range 51-60 years (33.3\%) followed by the age group of 61-70(26.66\%) and next 41-50 (25\%) (Table 1).

\begin{tabular}{|c|c|c|c|c|}
\hline $\begin{array}{l}\text { Age Group } \\
\text { (years) }\end{array}$ & Males & Females & $\begin{array}{l}\text { Total no of patients } \\
\qquad N=120\end{array}$ & $\begin{array}{c}\text { Percentage } \\
\text { (\%) }\end{array}$ \\
\hline $21-30$ & 1 & 1 & 2 & 1.6 \\
\hline $31-40$ & 1 & 5 & 6 & 5 \\
\hline $41-50$ & 20 & 10 & 30 & 25 \\
\hline $51-60$ & 14 & 26 & 40 & 33.3 \\
\hline $61-70$ & 12 & 20 & 32 & 26.66 \\
\hline $71-80$ & 6 & 4 & 10 & 8.33 \\
\hline Total & 54 & 66 & 120 & 100 \\
\hline
\end{tabular}

\section{Distribution of data based on gender}

Among the 120 patients involved in the study 54 (45\%) were males and $66(55 \%)$ were females (Figure 1).

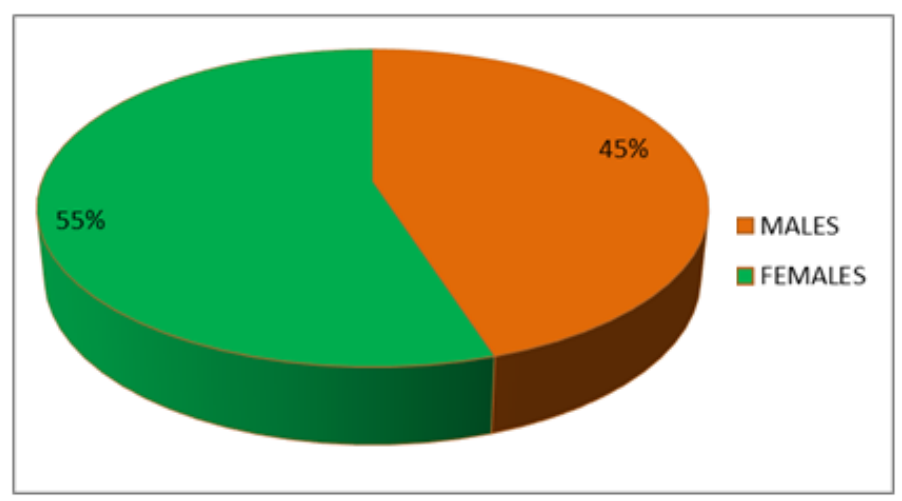

Figure 1: Sex distribution of the study population.

\section{Baseline Characteristics of Enrolled Subjects}

Among the 120 patients involved, the affected mean age group of the study population was $55.60 \pm 4.7$ years. Majority of the study subjects were females. Mean $\mathrm{HbA}_{1 \mathrm{c}}$ was $10.42 \pm 2.12 \%$. Duration of DM among these patients was $10.49 \pm 4.7$ years. Mean systolic BP was $121.19 \pm 22.8$ $\mathrm{mm}$ of $\mathrm{Hg}$ and diastolic BP was $80.78 \pm 10.45 \mathrm{~mm}$ of $\mathrm{Hg}$, Duration of fever was $2.1 \pm 1.67$ days and mean duration of hospital stay among these patients was $9.41 \pm 4.9$ days after starting antibiotic therapy (Table 2).

Table 2: Baseline characteristics of enrolled subjects.

\begin{tabular}{|c|c|}
\hline Parameters & Values \\
\hline Age $($ Mean \pm SD) & $55.60 \pm 11.46$ years \\
\hline Sex (Male/Female) & $54 / 66$ \\
\hline Duration of DM $($ Mean \pm SD) & $10.49 \pm 4.7$ years \\
\hline $\begin{array}{l}\text { Systolic BP } \\
(\text { Mean } \pm \text { sd) }\end{array}$ & $121.19 \pm 22.8 \mathrm{mmHg}$ \\
\hline $\begin{array}{l}\text { Diastolic BP } \\
(\text { Mean } \pm \text { sd) }\end{array}$ & $80.78 \pm 10.45 \mathrm{mmHg}$ \\
\hline $\begin{array}{c}\mathrm{Hba}_{1 \mathrm{c}}(\%) \\
(\mathrm{Mean} \pm \mathrm{SD})\end{array}$ & $10.40 \pm 2.12 \%$ \\
\hline $\begin{array}{l}\text { Duration of fever } \\
\quad(\text { Mean } \pm \text { SD })\end{array}$ & $2.1 \pm 1.67$ days \\
\hline $\begin{array}{c}\text { Duration of hospital stay } \\
(\text { Mean } \pm S D)\end{array}$ & $9.41 \pm 4.9$ days \\
\hline
\end{tabular}




\section{Clinical Manifestations}

Fever $(60.83 \%)$ was the most commonly observed symptom in the diabetes mellitus patients with urinary tract infections followed by vomiting (44.16\%), Dysuria (42\%), lower backache (19\%) and abdominal pain (29\%) (Figure 2).

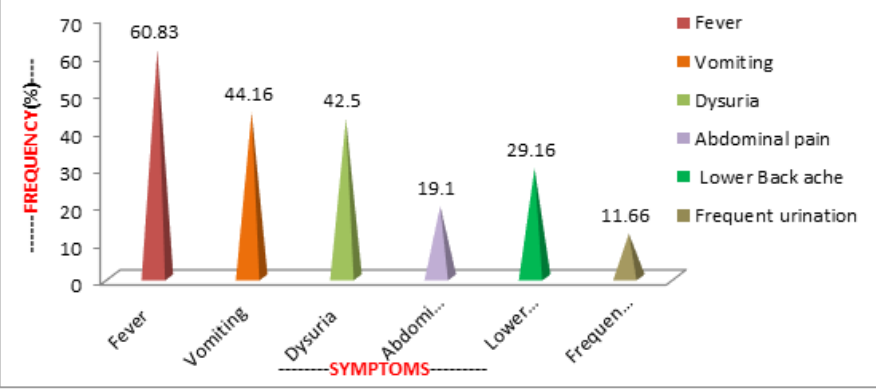

Figure 2: Frequency of Clinical Manifestations in type II DM patients with UTI.

\section{ISOLATED ORGANISMS FROM THE SAMPLE SIZE}

Among the organisms isolated, Escherichia coli (35\%) was the most common organism isolated followed by Enterococcus faecalis (17.6\%), Klebsiella pneumoniae (15.8\%), Proteus mirabilis (10.85\%), Staphylococcus aureus (9.16\%), Citrobacter (5.84\%) and 5.84\% of the study population did not show any bacterial growth. Among Gram-negative organisms isolated, Escherichia coli was the most commonly isolated organism followed by Klebsiella pneumoniae, Proteus mirabilis, Citrobacter. While Gram-positive organisms isolated include Enterococcus faecalis followed by Staphylococcus aureus (Figure 3).

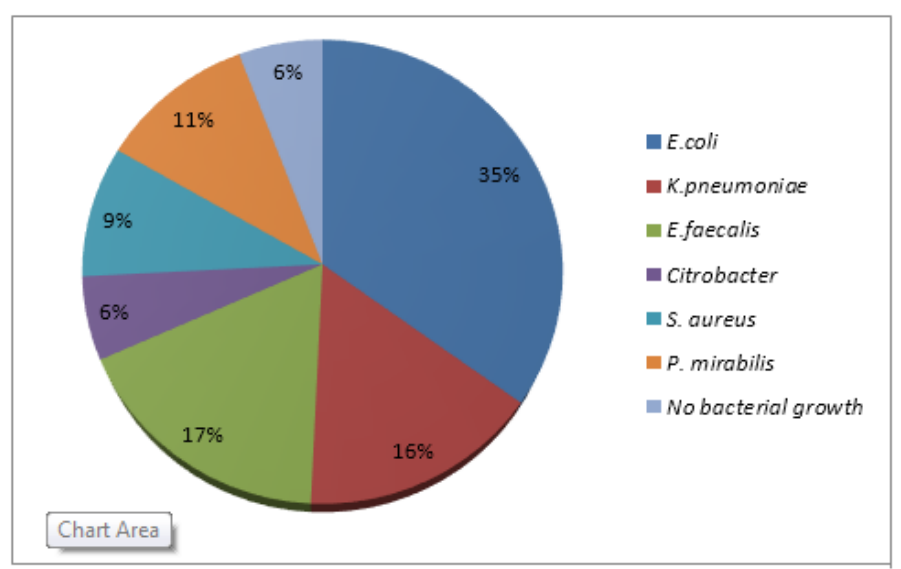

Figure 3: Isolated Organisms in type II DM with UTI patients.

\section{Data Evaluation of Antibiotic Sensitivity towards Micro- Organisms}

Data analysis showed that gram-negative organisms like Escherichia coli were highly sensitive to amikacin (90\%) followed by cefoperazone/ sulbactam (79\%). Klebsiella pneumoniae was sensitive to amoxicillin/ clavulanic acid (68\%) followed by cotrimoxazole (63\%). Citrobacter was sensitive to amikacin (71\%) followed by piperacillin/tazobactam (57\%), imipenem (57\%), gentamicin (57\%) and ciprofloxacin (57\%). Proteus mirabilis was sensitive to gentamicin (53\%) followed by cefoperazone/ sulbactam (46\%). Whereas gram-positive organisms like Enterococcus faecalis were sensitive to amoxicillin/clavulanic acid (76\%) followed by nitrofurantoin (52\%) and Staphylococcus aureus was sensitive to cotrimoxazole (45\%) followed by nitrofurantoin (40\%). (Table 3). Gramnegative organisms were sensitive to Amikacin (56.75\%), Cefoperazone/ sulbactam (53.5\%) and Piperacillin/tazobactam (46\%) (Figure 4) .While Gram-positive isolates were sensitive to Nitrofurantoin (46\%) followed by Amoxicillin/clavulanic acid (42.5\%) and Piperacillin/tazobactam (39.5\%) (Figure 5). Most uropathogens isolated in this study were sensitive to Amikacin (47.1\%) followed by Cefoperazone/sulbactam (46.5\%), Nitrofurantoin (44\%) and Piperacillin/tazobactam (43.8\%) (Figure 6).

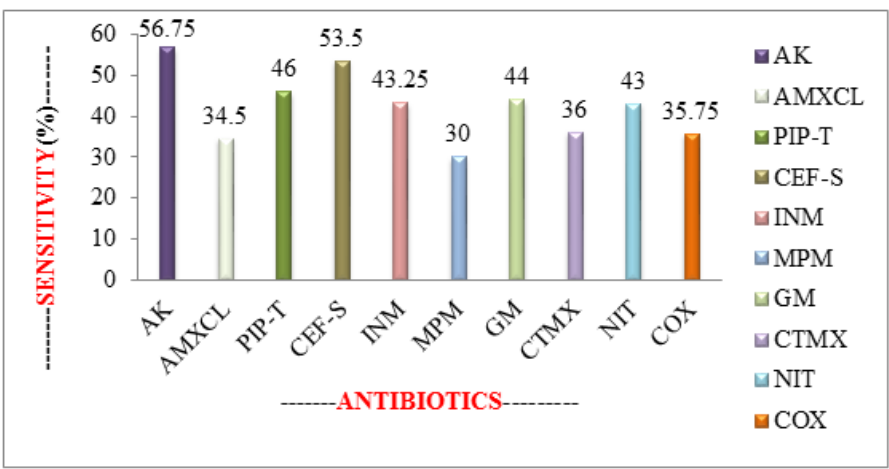

Figure 4: Overall Antibiotic sensitivity for Gram-negative Bacteria. Note:AK-Amikacin, AMXCL-Amoxycillin/clavulanicacid, PIP-T_Piperacillin/ tazobactam, CEFS-Cefoperazone/sulbactam, INM-Imepenem, MPMMeropenem, GM-Gentamicin, CTMX-Co-trimoxazole, NIT- Nitrofurantoin, COX-Ciprofloxacin

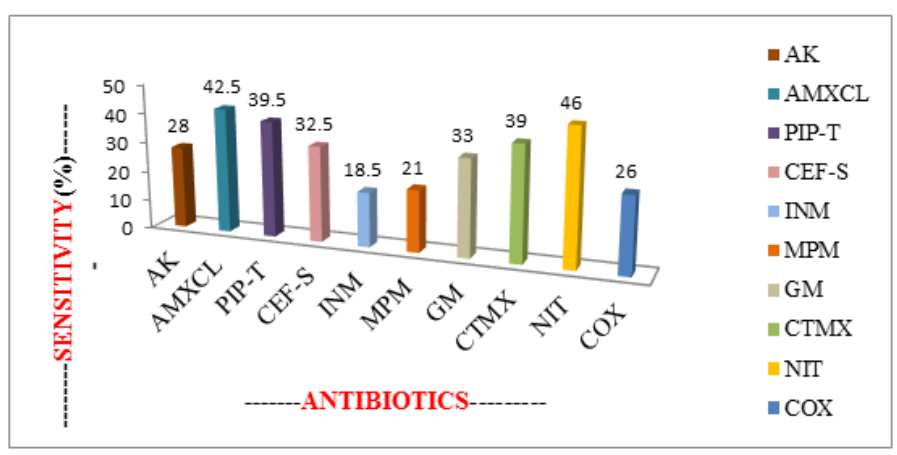

Figure 5: Overall Antibiotic sensitivity for Gram-positive Bacteria. Note: AK-Amikacin, AMXCL-Amoxycillin/clavulanicacid, PIP-T_Piperacillin/ tazobactam, CEFS-Cefoperazone/sulbactam, INM-Imepenem, MPMMeropenem, GM-Gentamicin, CTMX-Co-trimoxazole, NIT- Nitrofurantoin, COX-Ciprofloxacin.

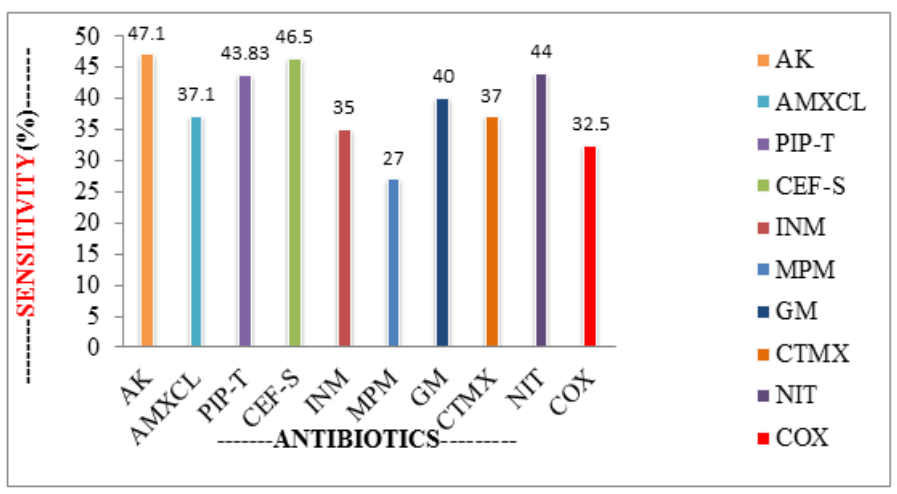

Figure 6: Overall sensitivity of commonly used Antibiotics. Note: AK-Amikacin, AMXCL-Amoxycillin/clavulanicacid, PIP-T_Piperacillin/ tazobactam, CEFS-Cefoperazone/sulbactam, INM-Imepenem, MPMMeropenem, GM-Gentamicin, CTMX-Co-trimoxazole, NIT- Nitrofurantoin, COX-Ciprofloxacin. 
Kuttiappan, et al.: Antibiotic Microbial Sensitivity in Type II Diabetes Mellitus Patients with Urinary Tract Infections

Table 3: Antibiotic Sensitivity towards Gram negative and Gram positive Bacteria.

\begin{tabular}{|c|c|c|c|c|c|c|}
\hline \multirow[t]{3}{*}{ Antibiotics } & \multicolumn{6}{|c|}{ Sensitivity (\%) } \\
\hline & \multicolumn{4}{|c|}{ Gram-negative bacteria } & \multicolumn{2}{|c|}{ Gram-positive bacteria } \\
\hline & E. coli & K. pneumoniae & Citrobacter & P. mirabilis & E. faecalis & S. aureus \\
\hline $\mathrm{AK}$ & 90 & 36 & 71 & 30 & 38 & 18 \\
\hline AMXCL & 19 & 68 & 28 & 23 & 76 & 9 \\
\hline PIP-T & 74 & 53 & 57 & 0 & 43 & 36 \\
\hline CEF-S & 79 & 47 & 42 & 46 & 38 & 27 \\
\hline INM & 43 & 42 & 57 & 31 & 10 & 27 \\
\hline MPM & 43 & 11 & 43 & 23 & 24 & 18 \\
\hline GM & 45 & 21 & 57 & 53 & 48 & 18 \\
\hline CTMX & 29 & 63 & 29 & 23 & 33 & 45 \\
\hline NIT & 67 & 50 & 40 & 15 & 52 & 40 \\
\hline $\operatorname{cox}$ & 29 & 42 & 57 & 15 & 43 & 9 \\
\hline
\end{tabular}

Note: AK-Amikacin, AMXCL-Amoxycillin/clavulanicacid, PIP-T-Piperacillin/tazobactam, CEFS-Cefoperazone/sulbactam, INM-Imepenem, MPM-Meropenem, GM-Gentamicin, CTMX-Co-trimoxazole, NIT- Nitrofurantoin, COX-Ciprofloxacin.

\section{DISCUSSION}

In this current study, we studied 120 diabetic patients to know the prevalence of various bacteria in diabetics with proven UTI and antibiotic sensitivity pattern. We found that females outnumbered males in terms of the prevalence of UTI. 51-60 years age group were most commonly affected with UTI among Type II diabetes mellitus patients. This was similar to an Indian study, ${ }^{9}$ a Nigerian study ${ }^{10}$ but it was different from a study in Sudan ${ }^{11}$ and East India. ${ }^{12}$

Most frequently affected patients belong to the age group $51-60$ years $(33.3 \%)$. This was similar to a study by R Simkhada ${ }^{13}$ but the study was different in Kuwait by May Sewify et al. ${ }^{14}$ and in Nepal by PK Jha et al. ${ }^{15}$ Like in almost all the studies from International or National locations ${ }^{16,17}$ the most common isolate was E. coli (35\%). It was similar to a casecontrolled study done in New Delhi ${ }^{18}$ which showed $E$. coli was the most common bacteria in UTI (64.3\%) whereas in a study from Nepal, ${ }^{13}$ it was $52.38 \%$ while in a Romania study ${ }^{19}$ it was $70.4 \%$.

In our study, we found K. pneumoniae (15.8\%), Proteus mirabilis (10.85\%) and Citrobacter (5.84\%) were the next common isolates amongst Gramnegative after E. coli. While E. faecalis (17.6\%) and S. aureus (9.16\%) were the most common Gram-positive isolates. E. coli was the most common isolate followed by E. faecalis which is same as seen in the study by Chatterjee et al ${ }^{14}$ If we observe the retrospective study in Nepal, there was a similar trend of the frequency of Enterococcus species (13.84\%), K. pneumoniae (8.3\%) and S. aureus (7.11\%). We also found Citrobacter species, Proteus species less frequently.

Most Gram-negative organisms were sensitive to Amikacin (56.75\%) followed by Cefoperazone-sulbactam (53.5\%) and Piperacillintazobactam (46\%).

Most Gram-positive organisms were sensitive to Nitrofurantoin (46\%) followed by Amoxicillin/clavulanic acid (42.5\%) and Piperacillin/ tazobactam (39.5\%). In our study, Nitrofurantoin had good activity against E.coli and Gram-positive isolates similar to as seen by PK Jha et al. ${ }^{12}$ Most uropathogens isolated in Type II DM were sensitive to Amikacin (47.1\%) followed by Cefoperazone/sulbactam (46.5\%), Nitrofurantoin (44\%) and Piperacillin/tazobactam (43.83\%).

\section{CONCLUSION}

In the current study it was concluded that E. coli, E. faecalis and $K$. pneumoniae were the most commonly isolated organisms in descending order and Citrobacter, Proteus mirabilis were less frequently isolated organisms. Most of the Gram-negative isolates were sensitive to Amikacin, Cefoperazone/sulbactam, Piperacillin/tazobactam and Nitrofurantoin, while Gram-positive isolates were sensitive to Nitrofurantoin followed by Amoxicillin/clavulanic acid and Piperacillin/ tazobactam. Most uropathogens isolated in this study were sensitive to Amikacin followed by Cefoperazone/sulbactam, Nitrofurantoin and Piperacillin/tazobactam were the good choices of antibiotics to start on empirical basis whereas Amikacin is a cost-effective injectable drug but its renal toxicity restricts its wide spread use.

This study recommended that these drugs should be the first-line drugs prescribed by clinicians while awaiting urine Microscopic culture sensitivity $(\mathrm{M} / \mathrm{C} / \mathrm{S})$ results in patients with UTI. Antibiotic sensitivity pattern of UTI pathogens should be periodically assessed to reduce the unnecessary use of antibiotics.

\section{ACKNOWLEDGEMENT}

We acknowledge entire health care Authorities for their appropriate response, along with the volunteers who big-heartedly provided support at the SVIMS hospital, Tirupati. The authors were grateful to Dr. Uma Maheswara Rao, Pharm.D Coordinator, SVIMS, Tirupati, Andhra Pradesh, India, for his continuous direction and care for this study.

\section{CONFLICT OF INTEREST}

The authors declare no conflicts of interest.

\section{REFERENCES}

1. Guariguata L, Whiting DR, Hambleton I, Beagley J, Linnenkamp U, Shaw JE. Global Estimation of diabetes prevalence for 2013 and projection for 2035 Diabetes Res Clin Pract. 2014;103(2):137-49.

2. Shaw JE, Sicree RA, Zimmet PZ. Global Estimation of diabetes for 2010 and 2030. Diabetes Res Clin Pract. 2010;87(1):4-14.

3. Whiting DR, Guariguata L, Weil C, Shaw J. Global Estimation of the prevalence of diabetes for 2011 and 2030. Diabetes Res Clin Pract. 2011;94(3):311-21.

4. Geerlings SE, Hoepelman AIM. Immune dysfunction in patients with diabetes mellitus (DM). FEMS Immunol Microb. 1999;26(3-4):259-65.

5. Kofteridis DP, Papadimitraki E, Mantadakis E, Maraki S, Papadakis JA, Tzifa G, et al. Effect of diabetes mellitus on the clinical and microbiological features of hospitalized elderly patients with acute pyelonephritis. J Am Geriatr Soc. 2009;57(11):2125-8.

6. Stamm WE. Measurement of pyuria and its relation to bacteriuria. Am J Med 1983;75(1):53-8. 
7. Ejrnaes K. Bacterial characterization of importance for recurrent urinary tract infections caused by Escherichia coli. Dan Med Bull. 2011;58(4):184-7.

8. Gupta S, Kapur S, Padmavathi D. Comparative prevalence of antimicrobial resistance in community-acquired urinary tract infection cases from representative states of Northern and Southern India. J Clin Diagn Res. 2014;8(9):09-12.

9. Viswanathan V, Janifer J, Geethalakshmi S, Satyavani K. Prevalence of lower urinary tract infection in South Indian type 2 diabetic subjects. Indian J Nephrol. 2009;19(3):107-11.

10. Ifediora $\mathrm{AC}$, Obeagu El, Akahara $\mathrm{Cl}$, Priscilla EU. Prevalence of urinary tract infection in diabetic patients attending umuahia health care facilities. J Bio Innov. 2016;5:68-82.

11. Hamdan HZ, Ziad AHM, Ali SK, Adam I. Epidemiology of urinary tract infections and antibiotics sensitivity among pregnant women at Khartoum North hospital. Ann Clin Microbiol Antimicrob. 2011;10(1):2.

12. Chatterjee N, Chatterjee C, Ghosh S, Mukhopadhyay M, Brahmachari R, Patar K. Pattern of Urinary Antibiograms in a Tertiary Care Hospital of Eastern India. J Assoc Physicians India. 2016;64(4):26-30.

13. Simkhada R. Urinary tract infection and antibiotic sensitivity pattern among diabetics. Nepal Med Coll J. 2013;15(1):1-4.

14. Sewify M, Nair S, Warsame S, Murad M, Alhubail A, Behbehani K et al. Prevalence of urinary tract infection and antimicrobial susceptibility among diabetic patients with controlled and uncontrolled glycaemia in Kuwait. J Diabetes Res. 2016;7

15. Jha PK, Baral R, Khanal B. Prevalence of Uropathogens in Diabetic Patients and their Susceptibility Pattern at a Tertiary Care Center in Nepal: A Retrospective Study. Int J Bio Lab Sci. 2014;3:29-34.

16. Baloch $\mathrm{GH}$, Jaffery MH, Madhudas C, Devrajaini BM, Ali SZ. Frequency and pattern in patients with diabetes mellitus. Prof Med J. 2011;18:466-9.

17. Chukwuocha UM, Emerole CO, Njokuobi TN, Nwawume IC. Urinary tract infections (UTIS) associated with diabetic patients in the Federal Medical Center, Owerri, Nigeria. Glob Adv Res J Microbiol. 2012;1 (5):62-6.

18. Goswami R, Bal CS, Tejaswi S, Punjabi GV, Kapil A, Kochupillai N. Prevalence of urinary tract infection and renal scars in patients with diabetes mellitus. Diabetes Res Clin Pract. 2001;53(3):181-6.

19. Chita T, Bogdan T, Muntean D, Luminița B, Horhat F, Hogea E, et al. Urinary tract infections in Romanian patients with diabetes: prevalence, etiology and risk factors. Ther Clin Risk Manag. 2016;13:1-7.

Article History: Submission Date : 24-12-2020; Revised Date : 09-01-2021; Acceptance Date : 11-02-2021

Cite this article: Kuttiappan A, Dondapati SP, Kothinti T, Bayyapureddy M, Kothapalli J, Balaji A. A Retrospective Study on Antibiotic Microbial Sensitivity in Type II Diabetes Mellitus Patients with Urinary Tract Infections. J Young Pharm. 2021;13(1):63-7. 